\title{
Nonrenewal spike trains generated by stochastic neuron models
}

\author{
Benjamin Lindner and André Longtin \\ Department of Physics, University of Ottawa, 150 Louis Pasteur, Ottawa, Canada KIN 6N5
}

\begin{abstract}
Many of the stochastic neuron models employed in the neurobiological literature generate renewal point processes, i.e., successive intervals between spikes are statistically uncorrelated. Recently, however, much experimental evidence for positive and negative correlations in the interspike interval (ISI) sequence of real neurons has been accumulated. It has been shown that these correlations can have implications for neuronal functions. We study an leaky integrate-and-fire (LIF) model with a dynamical threshold or an adaptation current both of which lead to negative correlations. Conditions are identified where these models are equivalent. The ISI statistics, the serial correlation coefficient, and the power spectrum of the spike train, are numerically investigated for various parameter sets.
\end{abstract}

\section{INTRODUCTION}

A common assumption made in many theoretical studies involving stochastic neurons, is the renewal property. Given a spiking neuron model that generates spike times at random instants $t_{i}$, this assumption implies that the interval sequence $I_{i}=t_{i}-t_{i-1}$ consists of entirely independent intervals. The serial correlation coefficient, defined by

$$
\rho_{j}=\frac{\left\langle\left(I_{i+j}-\left\langle I_{i}\right\rangle\right)\left(I_{i}-\left\langle I_{i}\right\rangle\right)\right\rangle}{\left\langle\left(I_{i}-\left\langle I_{i}\right\rangle\right)^{2}\right\rangle}
$$

is equal to $\delta_{j, 0}$ and the statistical properties of the spike train are entirely determined by the statistics of a single interval, i.e. by the probability density function (PDF) $P(I)$. This assumption is very convenient in particular when dealing with large networks of neurons (see, e.g., Ref. 1).

Much experimental evidence, however, has been accumulated in recent years that many neurons generate nonrenewal spike trains which display pronounced negative and/or positive correlations. One example are the P-units of weakly electric fish that display both positive and negative correlations in their ISI sequences. ${ }^{2,3}$ By computational studies it has recently been shown that negative correlations may lead to an enhancement of information transfer and detectability of weak signals ${ }^{3}$ (see also Ref. 4 for an analytical approach to the problem). On the other hand, positive correlations result in an optimal time scale for a detection task which shows up as a minimum in the Fano factor of the spike count vs counting time window. ${ }^{3,5}$

Basically, there are two different mechanisms that can give rise to correlations of ISIs. The first one is present in a model neuron stimulated by a strongly correlated input, for instance, a periodic stimulus or a colored noise. If the input signal is weak and slow, we expect that the ISI will depend in a linear fashion on the current value of the external stimulus. In this case the correlations of the input carry over to the ISI correlations, for instance, a weak and exponentially correlated noise with large correlation time will result in a positive exponential correlation among ISIs. ${ }^{5}$

The other mechanism relies on additional internal variables that are affected by the spiking of the neuron and in turn affect the spike generation mechanism. This can be seen as a feedback of a filtered version of the spike train. In this paper we will focus on this second mechanism by means of two different models. We show under which conditions these models are equivalent and study numerically their spike train and ISI statistics for various parameter sets. Analytical approaches to these systems will be detailed elsewhere. 


\section{MODELS AND QUANTITIES OF INTEREST}

The first model we shall study is a leaky integrate-and-fire model with a dynamical threshold ${ }^{3,6-8}$ (LIFDT, for short). The voltage in this model is governed by the usual linear equation for a white-noise driven leaky integrate-and-fire neuron, supplemented by the firing-and-reset rule as follows: whenever the voltage reaches the threshold $\Theta$, say at $t=t_{i}$, a spike is fired (firing time of the $\mathrm{i}$-th spike is thus $t_{i}$ ) and the voltage is reset to a value $v_{R}$. The threshold is itself a dynamical variable that decays exponentially toward a lower bound $\Theta_{0}$ between excitations but is increased by a constant amount at every spike. Suppose $i-1$ spikes have already been generated. Then the dynamics can be written as follows:

$$
\begin{aligned}
\dot{v}= & -v+\mu+\sqrt{2 D} \xi(t), \\
\dot{\Theta}= & -\frac{\left(\Theta-\Theta_{0}\right)}{\tau}+A \sum_{t_{j} \in \mathcal{T}} \delta\left(t-t_{j}\right), \\
& v(t)=\Theta(t) \Rightarrow t_{i} \doteq t, i \rightarrow i+1, \text { and } v\left(t^{+}\right)=v_{R},
\end{aligned}
$$

with $\tau$ being the characteristic time of decay of threshold toward its lower bound $\Theta_{0}$ and the last term in eq. (3) stands for rapid increases of the threshold after each firing.
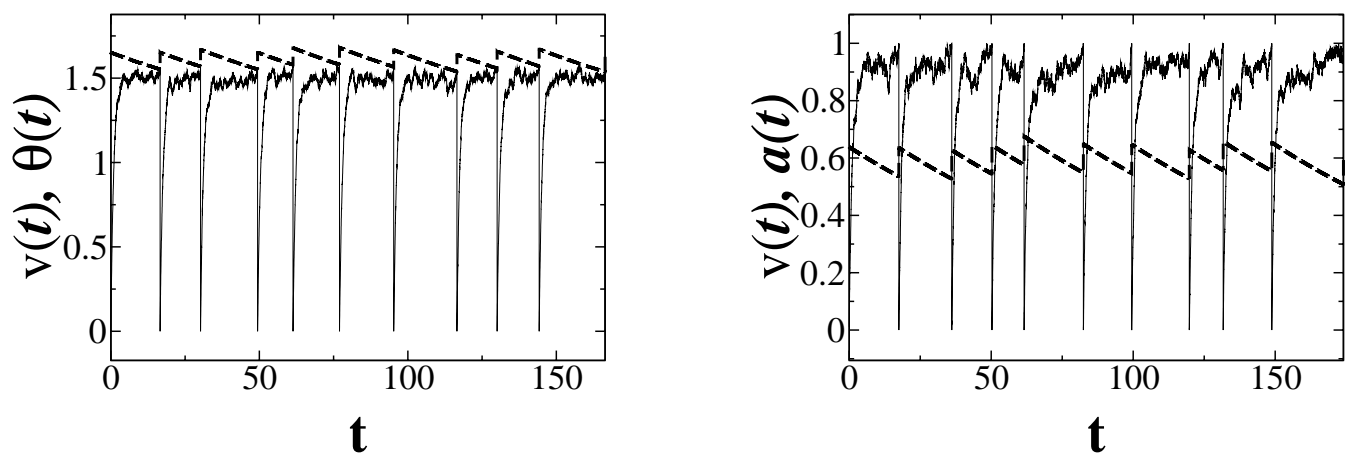

Figure 1. Trajectories of LIFDT model (left) and LIF model with adaptation current. Voltage variables are shown by solid lines, dynamical threshold (left) and adaptation variable (right) are indicated by dashed lines. Spikes occur at the instants of incrementation of $\Theta(t)$ or $a(t)$. Parameters in both models: $A=0.1, D=0.001, \mu=1.5, \tau=100$.

The second system is a white-noise driven LIF model with an adaptation current. This is a simplification of a conductance-based LIF model with a $\mathrm{Ca}^{2+}$ gated $\mathrm{K}^{+}$current $I_{A H P}$ (see, Ref. 7), i.e., we replace the time dependent conductance by a current. The dynamics for this model for the generation of the $i$-th spike is given by

$$
\begin{aligned}
\dot{v}= & -v+\mu-a+\sqrt{2 D} \xi(t) \\
\dot{a}= & -a / \tau+A \sum_{t_{j} \in \mathcal{T}} \delta\left(t-t_{j}\right) \\
& v(t)=\Theta_{0} \Rightarrow t_{i} \doteq t, i \rightarrow i+1, \text { and } v\left(t^{+}\right)=v_{R}
\end{aligned}
$$

where firings are generated with a constant threshold $\Theta_{0}$. The variable $a$ stands for the cytoplasmic $\mathrm{Ca}^{2+}$ concentration. Apart from an off-set, it is governed by the same dynamics as the dynamical threshold in the first model. The dynamics of both models are illustrated in Fig. 1.

It is possible to shift and rescale the variable $v$ in both models such that

$$
v_{R}=0, \quad \Theta_{0}=1 .
$$

These values are used throughout the following. 
Evidently, both models generate nonrenewal spike trains since the ISI depends on the respective additional variable $a$ or $\Theta$. Either models have been used in slightly different versions to model spike frequency adaptation. ${ }^{7,8}$ The LIFDT model with an additional periodic stimulation has been used to reproduce ISI statistics (including negative correlations among intervals) of P-type electroreceptors in weakly electric fish. ${ }^{3,6}$

Let us now consider quantities that characterize the ISI and spike train statistics. Given a spike train

$$
x=\sum_{t_{j} \in \mathcal{T}} \delta\left(t-t_{j}\right)
$$

generated by one of these models, we may construct a sequence of ISIs. From this sequence, we can estimate the probability density $P(I)$ of a single ISI, the mean ISI $\langle I\rangle$ and the coefficient of variation (CV) of the ISI

$$
C V=\sqrt{\left\langle I^{2}\right\rangle-\langle I\rangle^{2}} /\langle I\rangle
$$

as well as the serial correlation coefficient according to eq. (1). Another useful measure is the power spectrum of the spike train eq. (9), given by

$$
S(\omega)=\int_{-\infty}^{\infty} d \tau_{c} e^{i \omega \tau_{c}}\left\langle x(t) x\left(t+\tau_{c}\right)\right\rangle .
$$

A renewal spike train with the same statistics for a single ISI as in the original spike train can be obtained by shuffling the ISI sequence. We will also look at the power spectrum of this shuffled spike train in order to illustrate the effect of nonrenewal properties such as negative correlations on the power spectrum.

\section{RELATION BETWEEN LIFDT AND LIF WITH ADAPTATION CURRENT}

A relation between the models can be established by means of the transformation

$$
\tilde{v}=\lim _{\varepsilon \rightarrow 0^{+}} v(t) / \Theta_{\varepsilon}(t), \quad \Theta_{\varepsilon} \doteq \Theta(t-\varepsilon) .
$$

Applying this to the voltage variable of the LIFDT model leads to a model with constant threshold and reset values and the dynamics

$$
\begin{gathered}
\dot{v}=-v\left[1-\frac{1}{\tau}\left(1-\frac{1}{\Theta(t)}\right)\right]+\frac{\mu+\sqrt{2 D} \xi(t)}{\Theta(t)}-\lim _{\varepsilon \rightarrow 0} \frac{v}{\Theta_{\varepsilon}} A \sum \delta\left(t-\left(t_{j}+\varepsilon\right)\right), \\
\dot{\Theta}=-\frac{(\Theta-1)}{\tau}+A \sum_{t_{j} \in \mathcal{T}} \delta\left(t-t_{j}\right) \\
v(t)=1 \Rightarrow t_{i} \doteq t, i \rightarrow i+1, \text { and } v\left(t^{+}\right)=0
\end{gathered}
$$

where we have omitted the tilde and used eq. (8). The last term in the first line is zero (voltage is zero immediately after reset) and can be omitted. Thus the LIF with dynamical threshold is equivalent to a model with constant threshold but with leakage term and input current multiplying functions of the variable $\Theta(t)$. For small amplitude $A$, the leakage term will be just $-v$. This is evident for large $\tau$ but holds also to a good approximation if $\tau \sim 1$ since in this case the mean threshold will be close to $\Theta_{0}=1$. On the other hand, a weak amplitude $A$ also implies that the variable $\Theta$ will only weakly deviate from its stationary mean value $\langle\Theta\rangle$. Under these assumptions and using a new variable $a(t)=\Theta(t)-1$ the dynamics can be approximated by

$$
\begin{aligned}
\dot{v} & =-v+\left(1-\frac{\langle a\rangle^{2}}{(1+\langle a\rangle)^{2}}\right)(\mu+\sqrt{2 D} \xi(t))-a \frac{\mu+\sqrt{2 D} \xi(t)}{(1+\langle a\rangle)^{2}}, \\
\dot{a} & =-a / \tau+A \sum \delta\left(t-t_{i}\right) .
\end{aligned}
$$

This looks similar to the LIF with adaptation current, however, there are two differences: (i) base current and noise intensity as well as the variable $a$ in the voltage equation are rescaled by factors that depend on the 
stationary mean of $a$; (ii) the variable $a$ enters the voltage equation by a rescaling factor which is affected by the input noise. It is obvious that the factor multiplying base current and noise intensity is smaller than one. The factor multiplying the variable $a$ can be both smaller or larger than one, depending on the system's parameters. Both factors will be not too far from one for a base current about one, a weak amplitude $A$, and small up to moderate noise intensity. Under these conditions, we expect to find similar statistics for the LIFDT model and the LIF model with adaptation current.

\section{NUMERICAL RESULTS FOR THE LIFDT MODEL}

We have numerically integrated the model using a simple Euler procedure with a time step of $\Delta t=10^{-3}$. The first 100 spikes were discarded as transients, then the dynamics were simulated until $10^{5}$ spikes were obtained yielding reliable estimates of the spike and ISI statistics. In the following we show a sample trace of voltage and threshold, the power spectrum of the spike train, the ISI density, and the serial correlation coefficient as a function of lag. We kept the amplitude fixed at $A=0.1$ and varied the base current $\mu$, the noise intensity $D$, and the decay time of the second variable $\tau$. Regarding the choice of the base current $\mu$, it is important to distinguish subthreshold $\left(\mu<\Theta_{0}=1\right)$ and suprathreshold $\left(\mu>\Theta_{0}=1\right)$ values of $\mu$, qualitative differences for these two cases are expected especially in the weak-noise limit.

In Fig. 2 we show results for a suprathreshold base current $(\mu=1.5)$ at moderate noise $D=0.01$, and moderate decay time $\tau=1$, the latter being of the same order of magnitude as the time scale of the voltage variable as well as the mean ISI. Clearly, the threshold dynamics has only little effect on the spike statistics at these parameters. The ISI correlations are essentially zero, except for a very small negative value at lag one. The spike train power spectrum is peaked at the eigenfrequency and its higher harmonics as it is typical for a standard LIF driven by suprathreshold base current and white noise (see, Ref. 9). The spectrum of the shuffled ISI sequence is much the same as that for the original data indicating that correlations between intervals (also nonlinear ones not measured by $\rho_{l}$ ) do not effect the power spectrum. The ISI density is also peaked and looks pretty much like that of a standard LIF model. So for a decay time comparable to the ISI, the effect of a dynamical threshold is - at least for small amplitude $A$ - comparably weak.

Fig. 3 shows how the statistics change when choosing a decay time $\tau=100$ while keeping all other parameters unchanged. First of all, the mean ISI and the CV both considerably increase. A slower threshold is driven up to a higher stationary level as can be clearly seen in the panel depicting the trajectories. So the LIF dynamics sees a higher mean threshold and consequently generates larger and more variable ISIs. The shape of the ISI density is remarkable: its peak is widened and "topped off". Most interesting are the pronounced negative correlations found for $\rho_{l}$, in particular at the first lag. This non-renewal effect is clearly due to the dynamical threshold and implies that long ISIs are followed by short ones and vice versa. There are two effects of the threshold dynamics on the power spectrum of the spike train. First, the enlarged variability of the single ISI removes the pronounced peaks that were present for $\tau=1$ (or also for a standard LIF model). Second, the effect of negative correlations can be seen by comparison to the spectrum of the shuffled spike train which is renewal. The original data show less power at very small frequencies $(\omega<0.1)$ and slightly more power for $0.1<\omega<0.3$ than the spectrum of the shuffled spike train. We can understand the former result by a relation between the sum of correlation coefficients and the variability of spike count $n(t)$ given by Cox and Lewis. ${ }^{10}$ The variability of the spike count (i.e. its diffusion coefficient) in turn determines the power spectrum at zero frequency and one finds

$$
S(\omega=0)=\lim _{t \rightarrow \infty} \frac{\left\langle n(t)^{2}-\langle n(t)\rangle^{2}\right\rangle}{t}=\frac{C V^{2}}{\langle I\rangle}\left(1+2 \sum_{l=1}^{\infty} \rho_{l}\right)
$$

Hence, negative correlations lead to a decrease of power at low frequencies.

In Fig. 4 and Fig. 5 we show how the statistics change for $\tau=1$ and $\tau=100$ if we use a subthreshold base current $\mu=0.7$. In general, mean and variance of the ISI increase by decreasing the base current. For $\tau=1$, the trajectory of the threshold variable shows nearly always a complete decay to its lower bound $\Theta_{0}=1$ due to the much longer ISIs. For these parameters the spike train will be effectively a renewal process. Consequently, we observe again the typical statistics of a standard LIF model but with subthreshold base current, i.e. the ISI density is close to that of a Poison process with a high CV about 0.96, the power spectrum is almost flat, and 

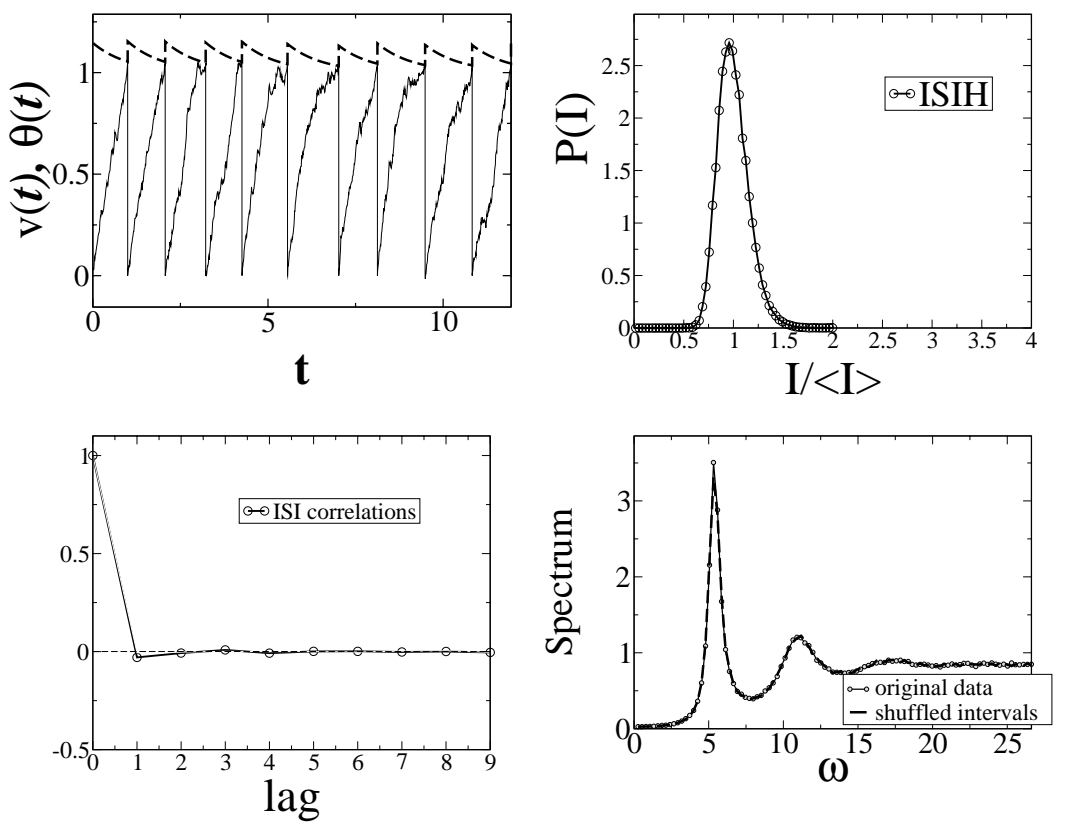

Figure 2. Results for the LIFDT model: trajectories, ISI density vs normalized interval (top left and right), serial correlation coefficient, and spectrum of spike train (bottom left and right) for the suprathreshold regime with $\mu=$ $1.5, D=0.01, \tau=1$. Mean and $\mathrm{CV}$ of the ISI were $\langle I\rangle=1.180$ and $C V=0.154$.
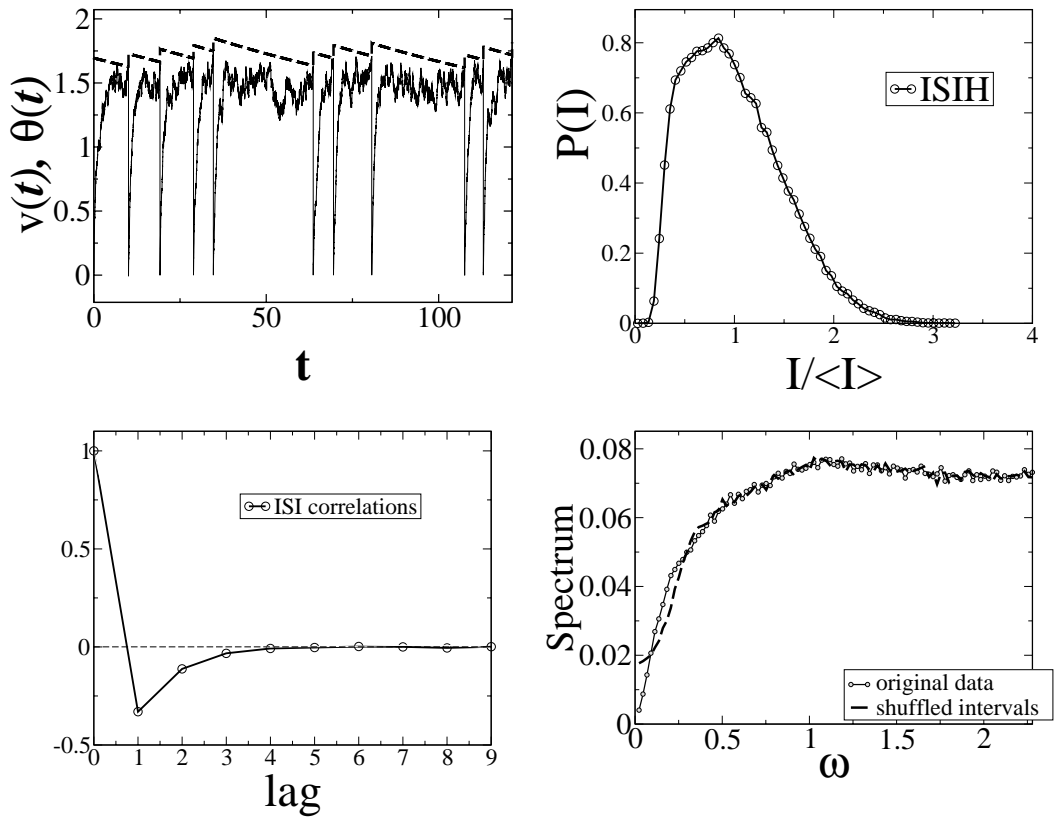

Figure 3. Results for the LIFDT model: trajectories, ISI density vs normalized interval (top left and right), serial correlation coefficient, and spectrum of spike train (bottom left and right) for the suprathreshold regime with $\mu=$ $1.5, D=0.01, \tau=100$. Mean and CV of the ISI were $\langle I\rangle=13.784$ and $C V=0.482$.

there are no correlations between ISIs $\left(\rho_{l} \approx 0\right.$ for $\left.l>0\right)$.

For $\tau=100$, the $C V$ decreases to a value $C V \approx 0.54$ that is surprisingly close to the one obtained for suprathreshold base current $(C V \approx 0.48)$, thus the threshold dynamics makes the variability largely independent of the base 

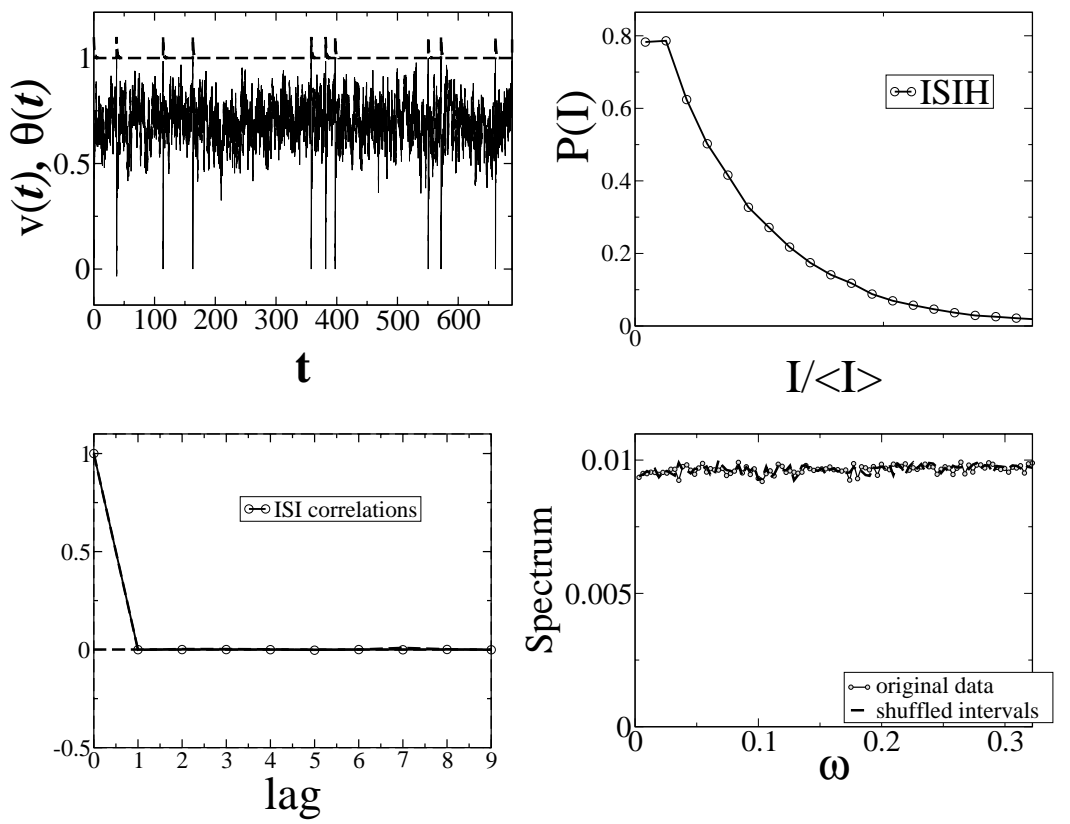

Figure 4. Results for the LIFDT model: trajectories, ISI density vs normalized interval (top left and right), serial correlation coefficient, and spectrum of spike train (bottom left and right) for the subthreshold regime with $\mu=0.7, D=$ $0.01, \tau=1$. Mean and CV of the ISI were $\langle I\rangle=97.5$ and $C V=0.965$.
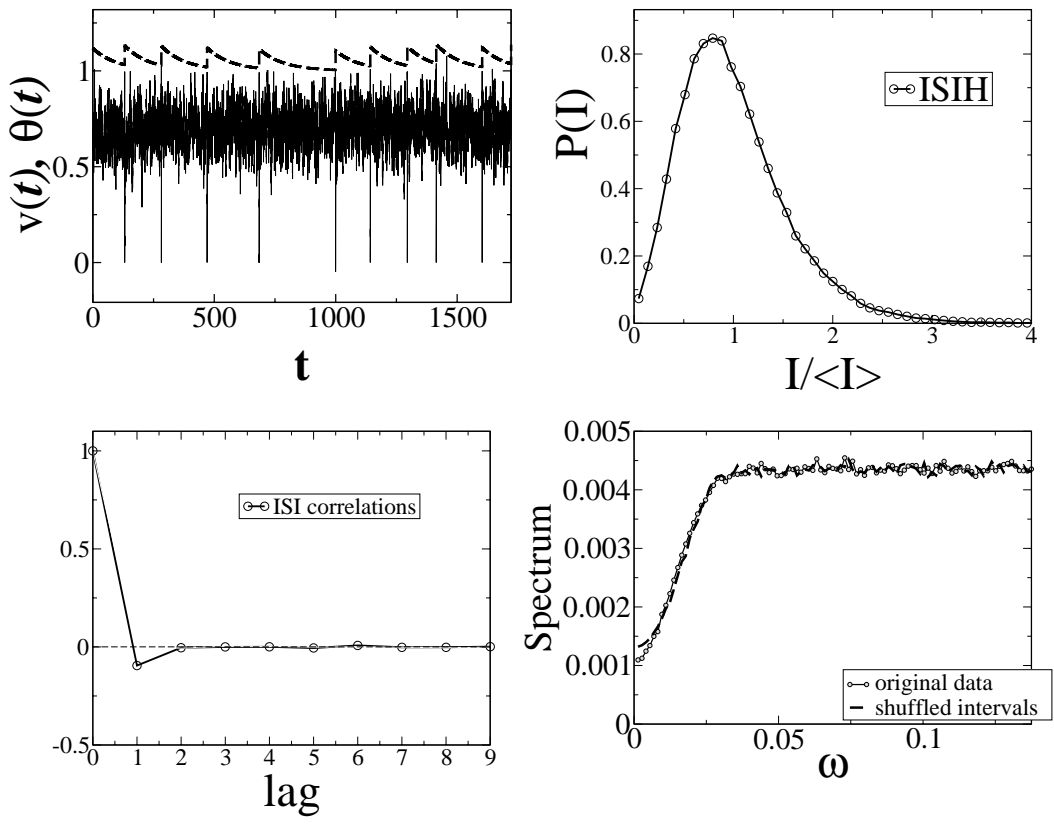

Figure 5. Results for the LIFDT model: trajectories, ISI density vs normalized interval (top left and right), serial correlation coefficient, and spectrum of spike train (bottom left and right) for the subthreshold regime with $\mu=0.7, D=$ $0.01, \tau=100$. Mean and CV of the ISI were $\langle I\rangle=228.5$ and $C V=0.542$.

current (note, however, that the mean ISI still differs considerably for $\mu=0.7$ and $\mu=1.5$ at $\tau=100$ ). From another point of view, one can also state that the threshold dynamics can decrease variability in the subthreshold regime whereas it increases the variability in the suprathreshold regime. Negative correlations are also present 

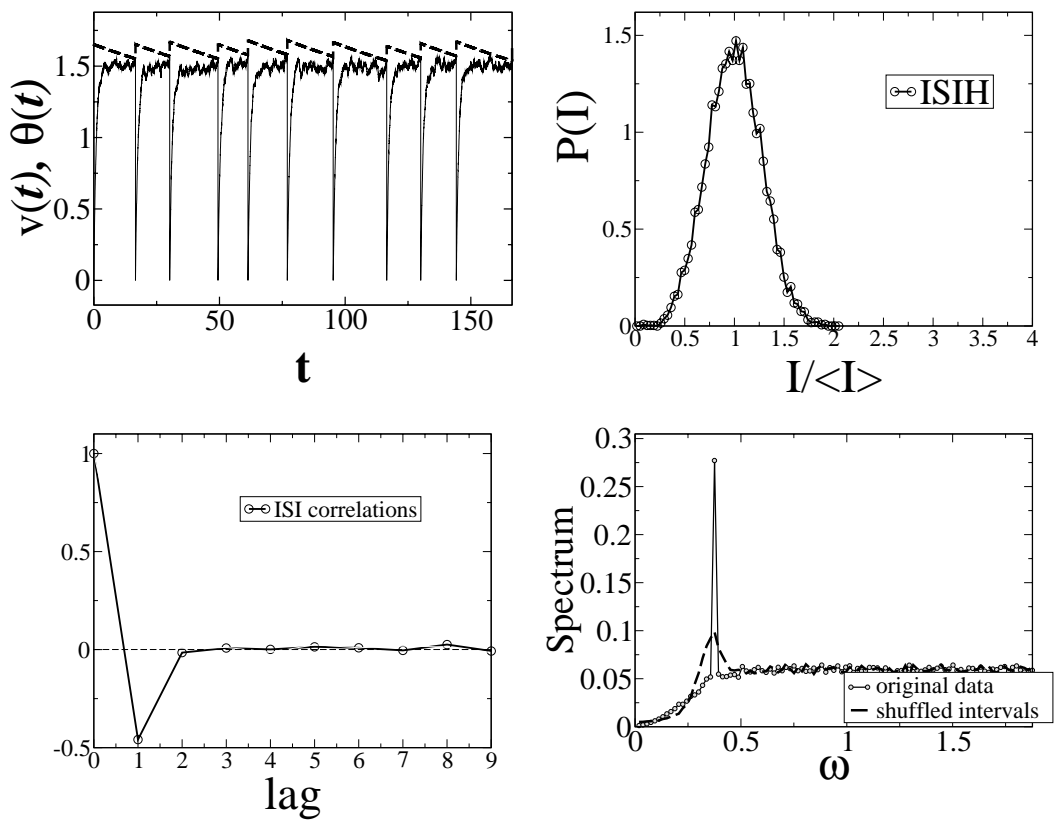

Figure 6. Results for the LIFDT model: trajectories, ISI density vs normalized interval (top left and right), serial correlation coefficient, and spectrum of spike train (bottom left and right) for the suprathreshold regime with $\mu=$ $1.5, D=0.001, \tau=100$. Mean and CV of the ISI were $\langle I\rangle=16.7$ and $C V=0.27$.
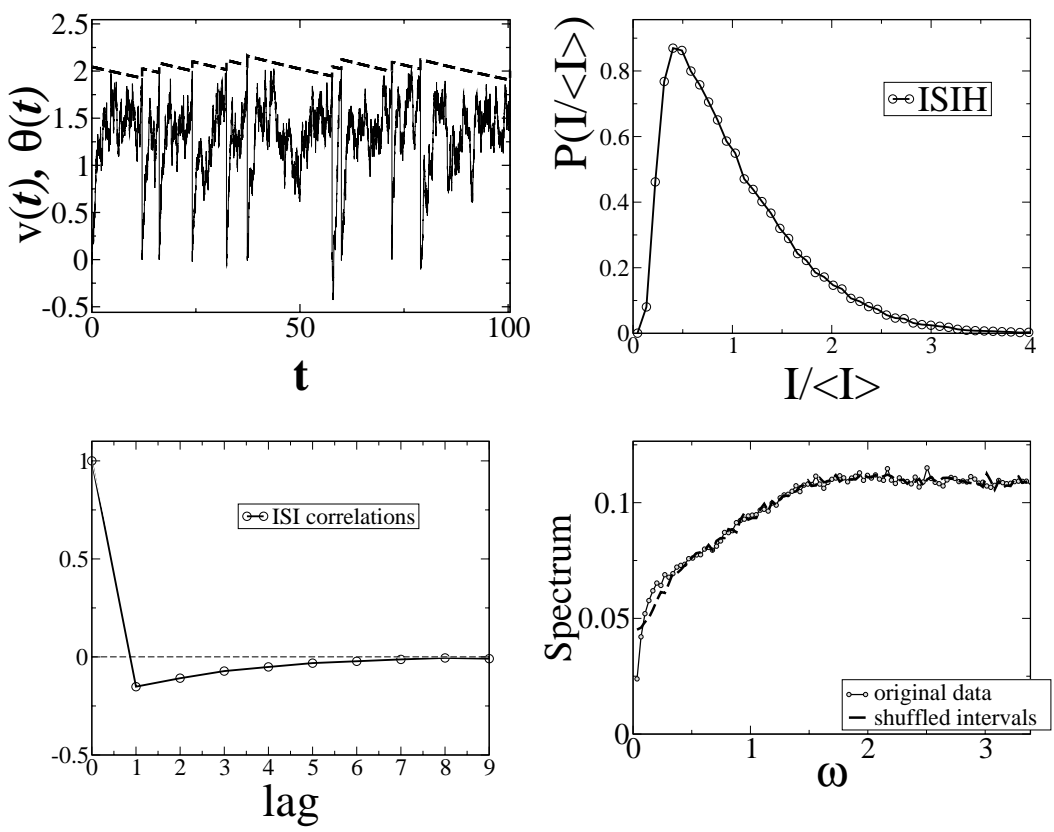

Figure 7. Results for the LIFDT model: trajectories, ISI density vs normalized interval (top left and right), serial correlation coefficient, and spectrum of spike train (bottom left and right) for the suprathreshold regime with $\mu=$ $1.5, D=0.1, \tau=100$. Mean and $\mathrm{CV}$ of the ISI were $\langle I\rangle=9.3$ and $C V=0.64$.

for $\tau=100$ at subthreshold base current, they are, however, slightly weaker and also restricted to the first lag compared to the corresponding suprathreshold case (for $\mu=1.5, \rho_{l}$ is also different from zero for lag 2 and 3 , cf. Fig. 3). Finally, the effect of these negative correlations on the power spectrum are similar to the suprathreshold 
case: the spectrum for the spike train with ISI correlations is smaller around $\omega=0$ than for the shuffled (renewal) spike train.

Turning back to the suprathreshold regime, we now ask how the statistics at large decay time change with the noise intensity. We fix base current and decay time at $\mu=1.5$ and $\tau=100$ and choose in Fig. 6 a lower and in Fig. 7 a higher noise intensity than in Fig. 3, respectively.

Clearly, a smaller noise intensity like $D=0.001$ as in Fig. 6 leads first of all to a larger mean ISI and to a lower ISI variability compared to Fig. 3 with $D=0.01$, as it would be also the case for a standard LIF model. The ISI density does not exhibit the broad maximum as for $D=0.01$. Remarkably, the negative correlation grows at lag 1 (it is close to -0.5 ) but gets closer to zero for higher lags compared to $D=0.01$. The pronounced negative correlation in the ISI sequence leads to a new feature in the power spectrum: the peak at the eigenfrequency of the spike generator is much higher than in the spectrum of the shuffled spike train. Hence, in the suprathreshold regime at weak noise, negative correlations lead to a strong periodic component or equivalently to a strong phase coherence. In order to illustrate phase coherence the reader may imagine the voltage started at its reset value at a certain instant: after a few intervals one may still be able to estimate the initial instant if the jitter of intervals is low. The time interval for which this estimation is yet feasible can be called a coherence length. A large coherence length will manifest itself by a high peak in the power spectrum (in fact, systems driven by a sinusoidally input possess an infinite coherence length and show consequently $\delta$ spikes in their power spectra). The negative correlations in the spike train will enlarge the coherence length: an ISI shorter than the mean ISI is on average followed by an ISI larger than the mean ISI; thus deviations from the mean ISI that result in losing phase coherence are "corrected" by the subsequent ISI. This mechanism works best for a negative correlation that is restricted to the first lag.

This is also confirmed by the results for large noise intensity $(D=0.1$, Fig. 7$)$ : small negative correlations become apparent at many lags slowly decaying with increasing lag. The effect of this correlation is pretty weak (weaker than for both $D=0.01$ and $D=0.001$ ) and restricted to very low frequencies. We also find a mean ISI smaller and a CV larger than for lower noise intensities as can be expected.

How do these noise-dependent features change if we use a subthreshold base current? No qualitative differences are expected at strong noise since in this case the dynamics does not depend much on the base current. The weak noise limit, however, will strongly differ, since even for a standard LIF with constant threshold, the rate decreases exponentially with the inverse noise intensity for $D \rightarrow 0$. When there is essentially no spiking in this limit, the dynamical threshold will be almost always close to its lower bound $\Theta_{0}=1$ and hence acts as a constant threshold. In other words, if the mean ISI for a standard LIF with constant threshold at $\Theta_{0}=1$ gets much larger than the decay time $\tau$ of the threshold dynamics, we expect a renewal spike train. Both predictions (no qualitative difference between the high-noise limits in sub- and suprathreshold regimes; renewal process in the weak-noise limit in subthreshold regime) are confirmed by our numerical simulation (not shown).

\section{NUMERICAL RESULTS FOR LIF WITH ADAPTATION CURRENT}

Adopting the parameters of the previous section for the second model we find for all parameter sets considered above the same qualitative behavior as for the LIFDT model. Even without a rescaling of the parameters, the ISI statistics, the serial correlation coefficient, and the power spectrum agree fairly well with those found for the LIFDT model at the same parameter sets. As an example we show data for the suprathreshold regime $(\mu=1.5)$ at large decay time $(\tau=100$ ), and weak (Fig. 8) and strong (Fig. 9) noise. These should be compared to Fig. 6 and Fig. 7, respectively.

For low noise we observe strong negative correlations indicated by a correlation coefficient at lag one that is close to -0.5 . Furthermore, we find a peak in the power spectrum that is much higher than in the spectrum of the shuffled spike train and only slightly lower than this in Fig. 6 . The only noticeable difference between the results for the models at these parameters is found at strong noise: in Fig. 7 we found a weak hump at $\omega \approx 2$ in the power spectrum which is absent in the spectrum of the LIF model with adaption current. A look at the spectrum of the shuffled spike train, however, reveals that this difference is rather related to the statistics of the single ISI than to the negative correlations induced by the adaptation current. The effect of negative correlations on the power spectrum is comparable to that of a dynamical threshold, that is a lowering of spectral power at low frequencies. 

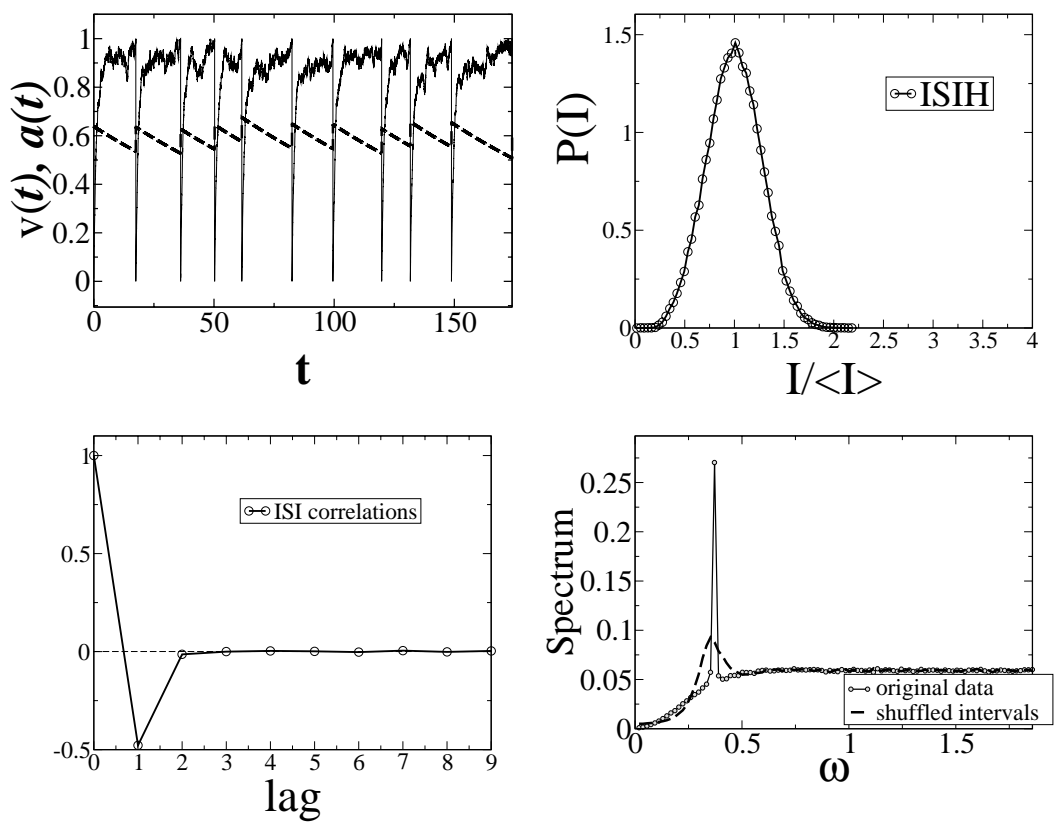

Figure 8. LIF with adaptation current: trajectories, ISI density vs normalized interval (top left and right), serial correlation coefficient, and spectrum of spike train (bottom left and right) for the suprathreshold regime with $\mu=$ $1.5, D=0.001, \tau=100$. Mean and CV of the ISI were $\langle I\rangle=16.9$ and $C V=0.275$.
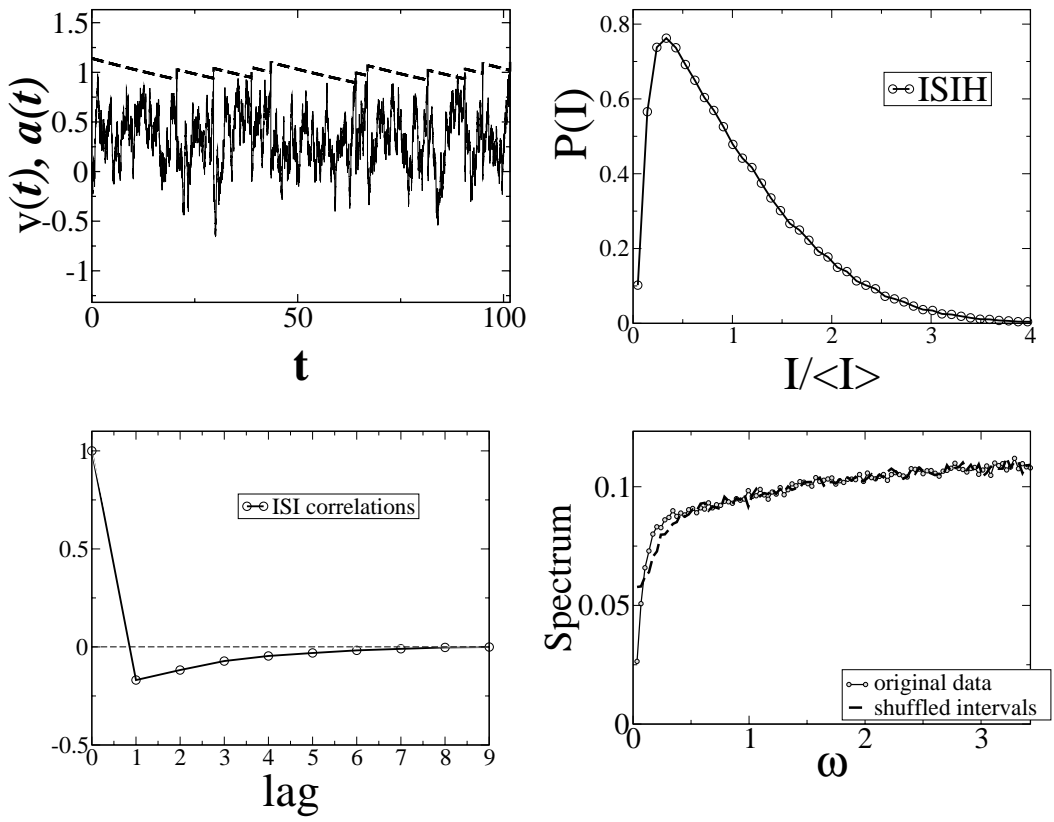

Figure 9. LIF with adaptation current: trajectories, ISI density vs normalized interval (top left and right), serial correlation coefficient, and spectrum of spike train (bottom left and right) for the suprathreshold regime with $\mu=$ $1.5, D=0.1, \tau=100$. Mean and CV of the ISI were $\langle I\rangle=9.2$ and $C V=0.72$.

\section{CONCLUSIONS}

We have studied two spiking neuron models that generate nonrenewal spike trains with pronounced negative correlations. It was shown that LIF models incorporating either a dynamical threshold or an adaptation current 
can generate spike trains with similar statistics when the coupling between the respective variable to the voltage (LIF) dynamics is weak and its typical time scale is large. It was furthermore demonstrated that the additional variable may decrease or increase the variability and the coherence of the ISI sequence and the corresponding spike train. There are two effects of negative correlations on the power spectrum: in general these correlations result in a decrease of spectral power at low frequencies; for strong enough correlations they can also strongly enhance the phase coherence of spiking, i.e. they sharpen the peak at the eigenfrequency of the neuron. In conclusion, we have shown that both models reveal a rich dynamical behavior that certainly deserves further investigations. An analytical treatment of the problem will be presented in a future publication.

\section{REFERENCES}

1. W. Gerstner, "Time structure of the activity in neural network models," Phys. Rev. E 51, p. 738, 1995.

2. R. Ratnam and M. E. Nelson, "Nonrenewal statistics of electrosensory afferent spike trains: Implications for the detection of weak sensory signals," J. Neurosci. 20, p. 6672, 2000.

3. M. J. Chacron, A. Longtin, M. St-Hilaire, and L. Maler, "Suprathreshold stochastic firing dynamics with memory in P-type electroreceptors," Phys. Rev. Lett. 85, p. 1576, 2000.

4. M. Chacron, B. Lindner, and A. Longtin, "Noise shaping by interval correlations increases neuronal information transfer," in preparation, 2003.

5. J. W. Middleton, M. Chacron, B. Lindner, and A. Longtin, "Firing statistics of a neuron model driven by long-correlated noise," submitted to Phys. Rev. E, 2003.

6. M. J. Chacron, A. Longtin, and L. Maler, "Negative interspike interval correlations increase the neuronal capacity for encoding time-dependent stimuli," J. Neurosci. 21, p. 5328, 2001.

7. Y.-H. Liu and X.-J. Wang, "Spike-frequency adaptation of a generalized leaky integrate-and-fire model neuron," J. Comp. Neurosci. 10, p. 25, 2001.

8. M. J. Chacron, K. Pakdaman, and A. Longtin, "Interspike interval correlations, memory, adaptation, and refractoriness in a leaky integrate-and-fire model with threshold fatigue," Neural Comp. 15, p. 253, 2003.

9. B. Lindner, L. Schimansky-Geier, and A. Longtin, "Maximizing spike train coherence or incoherence in the leaky integrate-and-fire model," Phys. Rev. E 66, p. 031916, 2002.

10. D. R. Cox and P. A. W. Lewis, The Statistical Analysis of Series of Events, Chapman and Hall, London, 1966 . 